\title{
Microstrip Triangular Loop Resonator Duplexer
}

\author{
A. Chinig, J. Zbitou, A. Errkik, A.Tribak, H. Bennis, and M. Latrach
}

\begin{abstract}
In this paper, a new microstrip duplexer topology is presented for Digital Communication System (DCS) at 1.8 GHz and for ISM Band at $2.45 \mathrm{GHz}$. A new approach for the design and the conception of the compact planar duplexer in microstrip technology is proposed. The suggested duplexer is based on two band pass filters (BPFs) using coupled microstrip triangular loop resonators. In this work Electromagnetic (EM) simulation with high meshing density and circuit modeling are achieved to meet some stringent performances concerning isolation, size, insertion loss and return loss.
\end{abstract}

Index Terms - Duplexer, triangular loop resonator, isolation, microstrip technology.

\section{INTRODUCTION}

Duplexers play a significant role in a wide variety of wireless communication systems and RF applications. Known as a tree-port device they allow to the transmitter and the receiver operating in different frequencies to use the common antenna [1]. The digital communication system (DCS) at $1800 \mathrm{MHz}$ band and the Industrial, Scientific and Medical (ISM) at $2.45 \mathrm{GHz}$ band, are very used in communication systems. Thus duplexers operating in 1800 $\mathrm{MHz} / 2.45 \mathrm{GHz}$ bands for DCS/ISM are desired to meet the corresponding IEEE standards. Duplexers based on microstrip filters are highly utilized in these applications due to their compact, planar, lightweight, and low-cost features.

The recent development in telecommunication technologies increased the demand for compact size, low cost and high performance duplexers. In this case several methods and structures to design a duplexer have been reported in literature such as parallel-coupled transmission lines [2], stepped impedance resonators [3, 4], miniaturized open-loop resonator [5] and balanced open-circuited periodic stubs [6]. The traditional method to achieve a microwave duplexer is to design two band pass filters with different frequencies and then to form the whole network. The insertion loss, return loss, isolation and frequency characteristic of the two BPFs are crucial parameters in the duplexer design.

In this paper, a novel duplexer design, based on triangular loop resonators is proposed for DCS and ISM applications. This circuit is the combination of two coupled band-pass.

The First section of this article describe the design of the new BPFs used in the duplexer, then a second section present the duplexer's design and the filters combination to reach the best results. Simulated frequency responses of the designed

Manuscript received February 14, 2013; revised April 17, 2013.

A. Chinig, J. Zbitou, A. Errkik, and H. Bennis are with the Laboratoire LITEN Faculté Polydisciplinaire, Khouribga, Morocco (email : Abdo.chinig@gmail.com).

A. Tribak is with the Institut national des postes et télécommunications, Rabat, Morocco.

M. Latrach is with the RF and Hyper group ESEO, Angers France. duplexer are also introduced. The circuit is developed in hybrid technology by using a substrate with a thickness of $1.58 \mathrm{~mm}$, a relative dielectric constant of 4.4 , a loss tangent of 0.01 , and conductor thickness of $35 \mathrm{um}$.

\section{A NARRow BAND PASS Filter Using TRIANGUlar LOOP RESONATOR}

Circular and square resonators have been used widely in the design of filters. However, thanks to their smaller size triangular microstrip loop was used in the design of filters [7]-[10].Based on the previous references a new band pass filter structure is introduced in this section. Fig. 1 and 2 show the structure of two BPFs using triangular loop resonators which the duplexer is based on.

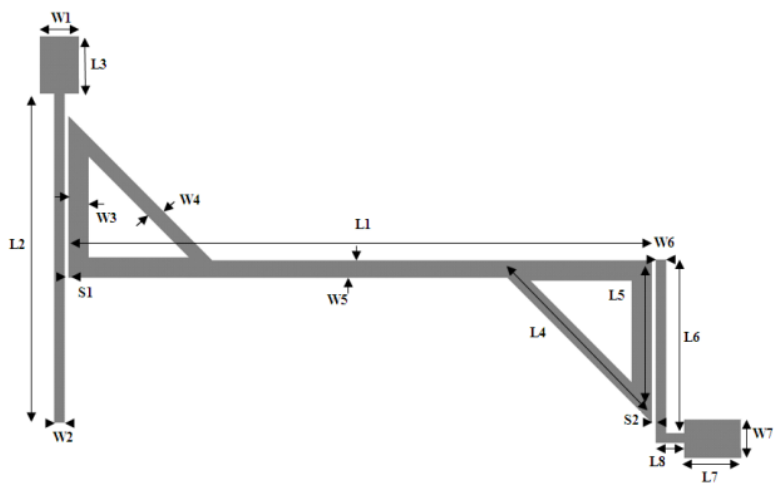

Fig. 1. Structure of the proposed filters for the $1.8 \mathrm{GHz}$ band.

The structural parameters as defined in Fig. 1 are: $L 1=$ 28.9, $L 2=16.3, L 3=2.8, L 4=11.31, L 5=8, L 6=8.56, L 7=$ $2.8, L 8=1.42, W 1=1.88, W 2=0.5, W 3=1, W 4=1, W 5=$ $0.86, W 6=0.5, W 7=1.88, S 1=0.2, S 2=0.2$, all dimensions are in $\mathrm{mm}$.

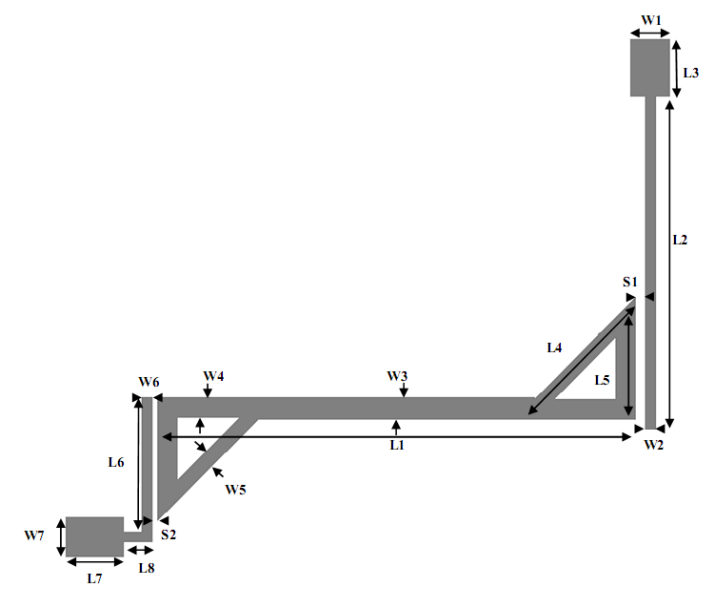

Fig. 2. Structure of the proposed filters for the $2.45 \mathrm{GHz}$ band.

The structural parameters as defined in Fig. 2 are: $L 1=$ 
23.42, $L 2=16.3, L 3=2.8, L 4=8.49, L 5=6, L 6=6.55, L 7=$ $2.8, L 8=1.42, W 1=1.88, W 2=0.5, W 3=1.05, W 4=0.99$, $W 5=1.01, W 6=0.5, W 7=1.88, S 1=0.45, S 2=0.25$, all dimensions are in $\mathrm{mm}$.

As can be seen from Fig. 1 and Fig. 2 each filter consists on a two coupled triangular resonators with two cross-coupled feed lines. The design of the pass filters is done at the center frequency of $1.8 \mathrm{GHz}$ for the lower channel and $2.45 \mathrm{GHz}$ for the higher one.

This filter was optimized and simulated by using Momentum electromagnetic simulator integrated into ADS 'Advanced Design System'. The Fig. 3 present the simulated performances obtained for both filters. From which, low insertion loss and high rejection can be clearly observed.

All dimensions $\mathrm{L}, \mathrm{W}$ and $\mathrm{S}$ were adjusted to get the desired response and to make the band pass match the high and the low bands. The band pass of the transmitting filter for the DCS is between 1.766 and $1.836 \mathrm{GHz}$, and for ISM the band pass of the receiving filter the is between 2.404 and 2.510 $\mathrm{GHz}$.

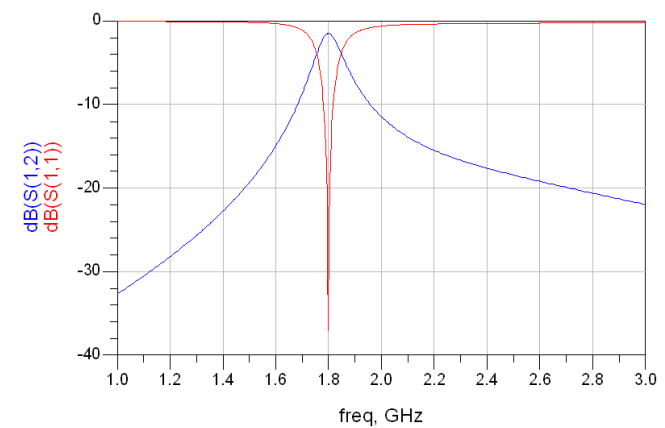

(a)

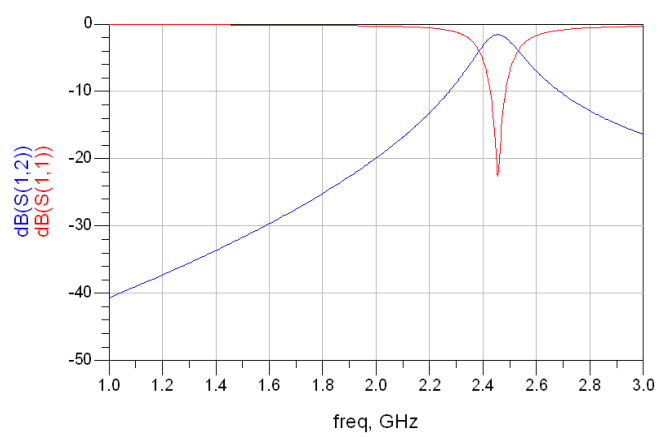

(b)

Fig. 3. Simulation results of the BPFs using triangular loop resonators (a) TX filter (b) RX filter.

Table I compares the simulated performances offered by the RX filter and the TX filter in terms of insertion losses, bandwidth and return loss.

TABLE I: SIMULATION RESULTS OF THE BPFS

\begin{tabular}{l||l||l}
\hline \hline \multirow{2}{*}{ Parameter } & \multicolumn{2}{|l}{ Simulation results } \\
\cline { 2 - 3 } & TX & RX \\
\hline \hline Frequency GHz & 2.456 & 1.799 \\
\hline \hline Bandwidth & $106 \mathrm{MHz}$ & $70 \mathrm{MHz}$ \\
\hline \hline Insertion loss $[\mathrm{db}]$ & 1.55 & 1.53 \\
\hline \hline Return loss $[\mathrm{db}]$ & 22.57 & 37.1 \\
\hline \hline
\end{tabular}

For the development of this new structure the substrate used was the FR4.

\section{DUPLEXER}

In the second step, a duplexer was designed based on the previous two BPFs used for ISM band at $2.45 \mathrm{GHz}$ and DCS band at $1.8 \mathrm{GHz}$.

Once the designed BPFs performances satisfy the considered communication systems, the main challenge that remains in this case is to interconnect the two filters without degrading the performance of the whole circuit. It was found that the diplexer performance might be distorted after combing the two individual BPFs even if the filter performance of the individual BPF satisfies the required communication systems for the DCS and ISM.

The idea was to use a simple feed line at the port 1 and the two resonators tuned to the desired band central frequencies. Each resonator is connected through cross coupling to a transmission line which is connected to an output port. The feed line is an essential part of the duplexer to join the both TX and RX filters without influence between each other.

Fig. 4 depicts the layout of a very compact duplexer designed with EM simulations. The dimensions of the circuit are $26 \mathrm{~mm}$ by $63 \mathrm{~mm}$.

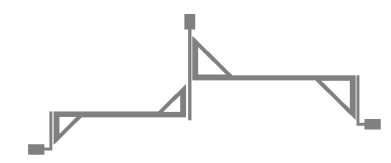

Fig. 4. Structure of the proposed duplexer.

The duplexer performance is simulated by using ADS, The simulation results for both the low and high bands are giving in Fig. 5.

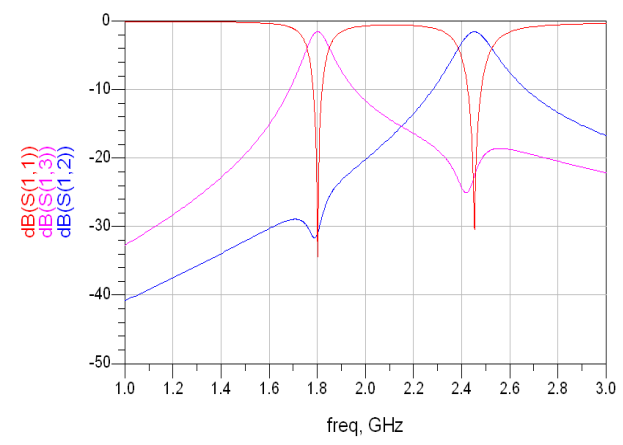

(a)

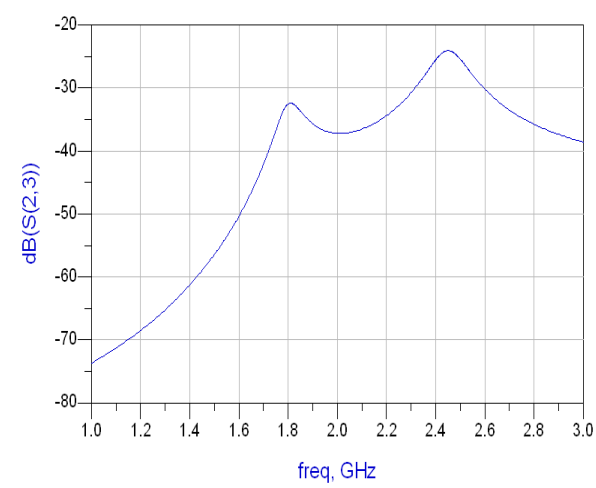

(b)

Fig. 5. Simulation results of the proposed duplexer (a) transmission response and return loss (b) Isolation. 
The band pass insertion loss is around $1.53 \mathrm{~dB}$ for low band remains lower than $1.6 \mathrm{~dB}$ for the high band. As shown in Fig. 5 the return loss are better than $-30.4 \mathrm{~dB}$ for transmission channel and $34.4 \mathrm{~dB}$ for the receive one. In addition the simulated isolation between the two channels is better than $24 \mathrm{db}$.

The observation shows that there is a small difference between the electrical response of the filters alone and the electrical response of the diplexer which represent weak coupling phenomena between the two filters.

The current distribution is simulated at $3 \mathrm{GHz}$ is shown in Fig. 6.

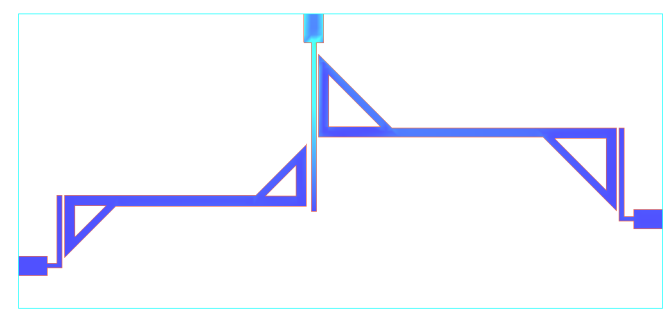

Fig. 6. Current distribution.

\section{CONCLUSION}

A new microstrip duplexer using triangular loop resonator composed of two BPFs coupled to the same microstrip feed line was proposed. The operation frequency of the duplexer is $1.8 \mathrm{GHz}$ at $\mathrm{RX}$ and $2.45 \mathrm{GHz}$ at TX. The results presented in this article show that the triangular loop resonator can be used to build a duplexer with a compact, a small size and a good electrical performance. The insertion loss of filters 1.54 $\mathrm{dB}$ and $1.57 \mathrm{~dB}$, for the return loss are $34.4 \mathrm{~dB}$ and $30.4 \mathrm{~dB}$ at RX and TX respectively. The isolation between the two channels is about $24 \mathrm{~dB}$ which shows that the simulation results in the band pass for the lower and upper bands are excellent for such kind of circuit.

\section{ACKNOWLEDGMENT}

We thank Mr. Mohamed Latrach Professor in ESEO, engineering institute in Angers, France, for allowing us to use all the equipments available in his laboratory.

\section{REFERENCES}

[1] T. F. Skaik, "Synthesis of coupled resonator circuits with multiple outputs using coupling matrix optimization," Ph.D. thesis, School of Electronic, Electrical and Computer Engineering, University of Birmingham, March 2011.

[2] G. Matthaei and E. G. Cristal, "Multiplexer channel-separating units using interdigital and parallel-coupled filters," IEEE Trans. Microwave Theory Tech., vol. 13, pp. 328-334, May 1965.

[3] T. Sugchai, "A design of wide-stopband microstrip diplexers with multiorder spurious-mode suppression using stepped-impedance resonators," IEEE Engineering and Technology (S-CET), 2012 Spring Congress, May 2012.

[4] D. Puttadilok, "A microstrip diplexer filter using stepped-impedance resonators," IEEE SICE Annual Conference, Aug. 2008.

[5] E. Goron, J. P. Coupez, C. Person, Y. Toutain, H. Lattard, and F. Perrot, "Accessing to UMTS filtering specifications using new microstrip miniaturized loop-filters," IEEE MTT-S Int. Microw. Symp. Dig., pp. 1599-1602, Jun. 2003.

[6] B. Strassner and K. Chang, "Wide-Band low-loss high-isolation microstrip periodic-stub diplexer for multiple-frequency applications," IEEE Trans. Microw. Theory Tech., vol. 49, pp. 1818-1820, 2001.
[7] C. Lugo and J. Papapolymerou, "Band pass filter design using a microstrip triangular loop resonator with dual-mode operation." IEEE Microwave and Wireless Components Letters, vol. 15, no. 7, July 2005

[8] R. B. Wu and S. Amari, "New triangular microstrip loop resonators for bandpass dual-mode filter application," IEEE MTT-S Int. Microw. Symp. Dig., 2005, pp. 941-944.

[9] A. GÄorÄur and C. Karpuz, "Cross-Coupled band pass filter using microstrip triangular open-loop resonators," in Proc. IEEE European Microwave Conference 2001, vol. 1-4, London, England, Oct. 2001.

[10] C. G. Sun, "A compact bandpass filter design using symmetrically triangular open loop resonator," presented in IEEE.

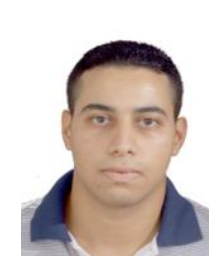

Abdessamed Chinig was born in Oued-Zem, Morocco, In 1988. He received the engineer's degree in electrica engineering from National School of Applied Sciences, Khouribga. University Hassan $1^{\text {er }}$ Morocco, and he is currently working toward the $\mathrm{Ph} . \mathrm{D}$. degree in communication engineering at Faculté des Sciences Techniques de Settat Morocco. His research interests include the analysis and design of microwave planar filters and duplexers.

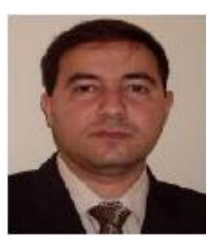

J. Zbitou was born in Fes, Morocco, in June 1976. He received the $\mathrm{Ph} . \mathrm{D}$. degree in electronics from the University of Nantes, Nantes, France, in 2005. He is currently a Professor of Electronics in FPK university Hassan $1^{\text {st }}$ Settat Morocco. He is involved in the design of hybrid, monolithic active, and passive microwave electronic circuits.

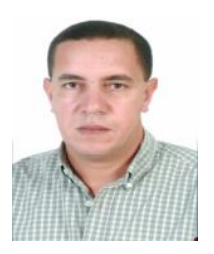

A. Errkik was born in July 1960 in Morocco. He received the Ph.D. degree in physics from the University of Technology Compiègne (UTC), Compiègne, France. $\mathrm{He}$ is currently a Professor of physics in FST university Hassan $1^{\text {st }}$ Settat Morocco. He is involved in the design of hybrid, monolithic active, and passive microwave electronic circuits

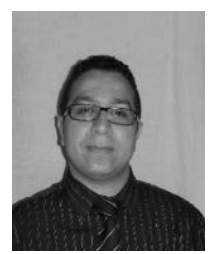

A. Tribak was born in Larache, Morocco, in 1981. He received the M.Sc. degree in physics from Abdelmalek Essaadi University, Tétouan, Morocco, in 2006. He received a Master degree in communications engineering from the University of Cantabria, Santander, Spain, in 2008, and received the $\mathrm{PhD}$ of Telecommunication degree in 2011, from the University of Cantabria, Santander, Spain. Since 2006 to 2011, he has been with the Department of Communications Engineering, University of Cantabria. Since 2011 he is a Professor in the National Institute of Poste and Telecommunication, Rabat, Morocco. His main area of activities is microwave circuits and systems; antenna feed subsystems for satellite and radio-astronomy applications.

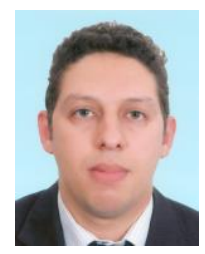

H. Bennis was born in Meknes, Morocco, in September 1977. He received the Ph.D. degree in Computer Science and Telecommunication from the University of Mohammed V Agdal, Rabat, Morocco, in 2011. He is currently a Professor of Computer Science and Computing Network in FPK University Hassan $1{ }^{\text {st }}$ Settat Morocco. He is involved in the design of hybrid, monolithic active and passive microwave electronic circuits

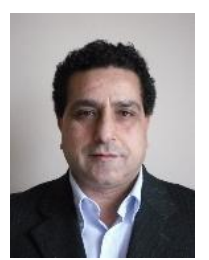

M. Latrach was born in Douar Ksiba, Sless, Morocco, in 1958. He received the Ph.D. degree in electronics from the University of Limoges, Limoges, France, in 1990. He is currently a Professor of microwave engineering with the Ecole Suprieure d'Electronique de l'Ouest (ESEO), Angers, France, where his research involves RF and microwaves. His field of interest is the design of hybrid, monolithic active, and passive microwave circuits, metamaterials, LH materials, antennas and their applications in wireless communications, and wireless power transmission 